\title{
Análise por Pontos de Função: Uma Técnica para Dimensionamento de Sistemas de Informação
}

\author{
Raquel Dias \\ Instituto de Cooperação e Assistência Técnica da AEUDF - Av. W4 Sul - Q 704 - Brasília - DF \\ raquel dias@directnet.com.br
}

\begin{abstract}
Resumo - Este artigo aborda a técnica de Análise por Ponto de Função (APF) como ferramenta adequada para auxiliar no processo de gestão de Tecnologia da Informação (TI). Gerentes de $\mathrm{TI}$ podem medir a produtividade relacionada com o esforço realizado no processo de desenvolvimento, otimização e manutenção de sistemas de informação. Através desta técnica é possível calcular o esforço despendido por unidade, ou por atividade no departamento de TI, independente do tipo do sistema, da tecnologia utilizada para desenvolver o produto, e da habilidade dos desenvolvedores, e ainda, fornecer subsídios para melhor compreensão das correções, falhas e dos problemas de planejamento dos projetos já concluídos ou em andamento. Permite criar ambiente padrão na área de TI com possibilidade de comparação de seu desempenho com o mercado.
\end{abstract}

Palavras-chave: Análise por ponto de função, gestão de tecnologia da Informação, métricas.

\begin{abstract}
This article approaches the technique of Function Point Analysis (PFA) as adjusted tool to assist in the management process of Information Technology (IT). IT managers can measure the productivity related with the effort carried through in the development, otimization and maintenance process. Through this technique it is possible to calculate the effort expended for unit, or activity in the TI department, independent of the system type, the used technology to develop the product, and of the ability of the developers, and still, to supply subsidies better understanding of the corrections, imperfections and of the problems of planning of the finished projects or in progress. It allows to create TI standard enviroment and the possibility of comparison of its performance with the market .
\end{abstract}

Key-words: Function Point Analysis (PFA), Information technology management, metric.

\section{Introdução}

A evolução do uso da informação no mundo dos negócios não pode mais ser ignorada pois tornouse fator determinante de competitividade. Empresas em todos os ramos de atividades passaram a se preocupar em buscar respostas para perguntas como: estamos usando adequadamente a informação? possuímos suficientes recursos tecnológicos? qual deve ser a estratégia de investimentos em Tecnologia da Informação (TI) em nossa empresa?

Na gestão de TI o maior problema consiste em se definir indicadores para o gerenciamento dos recursos para o atendimento pleno da demanda dos usuários com presteza e qualidade.

A demanda por produtos e serviços de informática cresce continuamente em função da necessidade de novos serviços, suportes e manutenções legais, enquanto o orçamento da área sofre reduções, sistemáticas, a cada plano de governo, ou a cada política, de enxugamento e/ou contenção de despesas, implementada.

Selecionar métricas adequadas para 0 gerenciamento de TI não é uma tarefa trivial, principalmente devido à natureza dinâmica da área. A complexidade aumenta quando observamos a existência de considerável participação das despesas com recursos humanos na execução orçamentária. Como gerenciar os recursos que se reduzem a cada ano, para que se possa atender um número cada vez maior de usuários? Quais as métricas de desempenho de sistemas são mais usadas? Qual o grau de satisfação de nossos clientes? Estas e outras perguntas são freqüentes na área de tecnologia da informação, e a busca por respostas constitui-se tarefa árdua para os administradores que, na maioria das vezes, são obrigados a assumir compromissos com usuários sem a certeza de dispor de recursos necessários para o atendimento.

A técnica de análise por Ponto de Função auxilia os gerentes $\mathrm{Tl}$ a medir a produtividade relacionada com o esforço realizado no processo de desenvolvimento, otimização e manutenção de sistemas. Baseia-se em três taxas de produtividade de sistemas - distribuição, modificação e suporte - todas consideradas sob o ponto de vista do usuário. Através desta técnica é possível calcular o esforço despendido por unidade, ou por atividade no departamento de TI e ainda, fornecer subsídios para melhor compreensão das correções, falhas e dos 
problemas de planejamento dos projetos já concluídos ou em andamento, de forma a desenvolver projetos futuros de maneira mais eficiente.

A taxa de produtividade pode ser entendida como a razão entre a quantidade de produtos liberados e o esforço despendido no desenvolvimento, expressa em um período de tempo. Para identificar oportunidades de melhoria, há que se conhecer a taxa atual de produtividade das unidades produtoras de forma a comparar com a meta definida. Por exemplo, na indústria automobilística, a saída é facilmente mensurada a partir da contagem de carros que saem da linha de produção. Porque cada carro é o mesmo e os meios pelos quais eles são manufaturados, e as atividades, executadas por cada trabalhador na linha de produção, são padronizadas, desta forma o método de contagem é válido. No caso de sistemas de informação, entretanto, cada aplicação é diferente, o processo através do qual cada aplicação é desenvolvida varia, e as atividades executadas são significativamente afetadas pelas habilidades dos trabalhadores. Devido a estas variáveis este processo torna-se muito complexo, invalidando a utilização do método de contagem abordado em uma indústria automobilística. Este enfoque de contagem (ex. considerar o número de sistemas implementados por mês) não é apropriado para medir a quantidade de saída ou produto. Para o departamento de informática a produtividade deve ser medida através de um método que seja independente do tipo do sistema, da tecnologia utilizada para desenvolver o produto, e da habilidade dos desenvolvedores. A técnica de pontos de função - desenvolvida por Allan Albrecht da IBM - fornece o método que mais se aproxima do atendimento aos requisitos mencionados. [1]

\section{Significado e Propósito dos Pontos de Função}

Não considerando a tecnologia utilizada, para o desenvolvimento de um sistema de informação e o ambiente no qual ele será executado, cada sistema tem como objetivo comum - executar um conjunto definido de ações/funções para o usuário final. A técnica de pontos de função faz a medição de um sistema baseado nas características relativas ao seu funcionamento e objetivos identificadas a partir da visão do usuário. Assim, como o dimensionamento do sistema é considerado sob o ponto de vista do usuário, o processo envolve a capacidade funcional do sistema que está a disposição do usuário, não dependendo da tecnologia empregada para o desenvolvimento, ou do conhecimento, habilidade e experiência do grupo que desenvolveu ou fez a manutenção do sistema.
Devido a esta medição, tecnologicamente independente, comparando o tamanho dos sistemas, dimensionados em pontos de função, os gerentes de informática podem comparar os diversos sistemas. Combinando o tamanho dos pontos de função com muitas outras métricas, pode-se medir os efeitos e o desempenho das unidades, e definir plano de ações voltado para o atendimento das várias metas de produtividade.

\section{Metodologia}

Trata-se de uma pesquisa, exploratória. O objetivo principal foi o aprimoramento de idéias e a descoberta de intuições. Envolveu, portanto, levantamento bibliográfico, análise de dados e observações coletadas com a experiência resultante durante a aplicação prática da Técnica de análise de pontos de função em sistemas da Caixa Econômica Federal.

O levantamento bibliográfico concentrou-se basicamente na leitura dos manuais e de artigos relativos à técnica. O resultado culminou com a elaboração de recomendações para aqueles que desejam mensurar o portifólio de sistemas utilizados em sua empresa.

\section{Fundamentação Teórica - Primeiras métricas [1]} Linhas de Códigos

- Dimensionamento dos sistemas através da quantidade de linhas de códigos.

Apresenta dificuldades para uso em comparações de sistemas desenvolvidos por linguagens diferentes;apresenta distorções, para efeito de comparação, por ser dependente do estilo dos programadores; apresenta impossibilidade de uso em estimativas, considerando que os sistemas somente poderiam ser medidos depois de codificados.

\section{Sistema Halstead}

- Dimensionamento dos sistemas baseado na quantidade de operandos (itens de dados) e operados (comandos de linguagem de dados)

Apresenta dificuldades por ser dependente das características da linguagem de programação; dependente do estilo dos programadores; apresenta dificuldades para uso em estimativas, considerando que os programas somente poderão ser medidos após codificados.

\section{Análise por Pontos de função [1] [2] [3]}

Mede a funcionalidade do sistema baseado na visão do usuário, apresentando as seguintes características:

口 independência de tecnologia utilizada. 
- Produção de resultados consistentes.

- Baseada na visão do usuário.

․ Significância para o usuário final.

口 Utilização em estimativas.

- Passível de automação.

Apresenta dificuldades por possuir relativa subjetividade, considerando que reflete a visão do usuário. A complexidade está relacionada com volumes de arquivos lógicos, registros lógicos e itens de dados, identificados. Considera 14 Itens de influência subdivididos em subitens que abrangem todos os aspectos relacionados às necessidades de uso de ferramentas para atendimento das requisições dos usuários.

\begin{tabular}{|l|l|l|l|}
\hline Características & $\begin{array}{l}\text { Linhas de } \\
\text { Código }\end{array}$ & $\begin{array}{l}\text { Sistema } \\
\text { Halstead }\end{array}$ & $\begin{array}{l}\text { Pontos } \\
\text { de } \\
\text { Função }\end{array}$ \\
\hline Independência de tecnologia & Não & Não & Sim \\
\hline Resultados consistentes & Sim & Sim & Sim \\
\hline Visão do usuário & Não & Não & Sim \\
\hline Significância para o Usuário final & Não & Não & Sim \\
\hline Utilização em estimativas & Não & Não & Sim \\
\hline Passível de automação & Sim & Sim & Sim \\
\hline
\end{tabular}

Tabela 1 : comparação das técnicas apresentadas

\section{Visão Geral da Análise de Pontos de Função [1] [2] [3]}

Trata-se de técnica que permite medir a funcionalidade de um software ou aplicativo, sob a visão do usuário, e a partir da descrição dos requisitos do usuário.

Objetivos da técnica:

- Medir a funcionalidade dos sistemas independentemente da tecnologia que foi utilizada no seu desenvolvimento;

- Avaliar com base no que o sistema faz, ou seja, as funções que o sistema executa, independente da tecnologia em que foi implementado;

- Identificar um padrão de medida para a produtividade e qualidade

- Fornecer ferramenta para auxiliar nas estimativas de desenvolvimento de software;

a Possibilitar o uso por diferentes projetos, em diferentes empresas e ambientes variados, demonstrando consistência na comparação;

- Ser compreensível pelo pessoal não técnico;

口 Ser utilizável em todo o ciclo de desenvolvimento do software;

- Ser um processo simples de mensuração;

a Ser conciso e consistente.

\section{Benefícios com o Uso da APF}

a Dimensionamento dos sistemas, em produção e/ou em desenvolvimento bem como, solicitações de manutenção.

- Apoio para estimativa de custos e recursos requeridos para $o$ desenvolvimento $\mathrm{e}$ manutenção de software.

- Apoio para gerenciamento da qualidade e produtividade no processo de desenvolvimento do software.
- Apoio para a tomada de decisão relativa a seleção para aquisição de pacotes, e contratação de serviços.

\section{Etapas para Avaliação - Cinco Etapas}

\section{Etapa I Identificação do Tipo de Contagem a ser Utilizado}

O quê vou medir? Consiste na identificação do objeto a ser medido, como sendo um projeto de desenvolvimento, manutenção ou produção.

\section{Etapa II Definição da Fronteira da Aplicação}

Quais os limites do que vou medir? esta é a etapa em que é estabelecido o escopo do sistema objeto da avaliação, sob a visão do usuário. Neste momento são identificados todos os relacionamentos do sistema, sob avaliação, com o seu exterior, e são identificadas as pertinências dos dados e os processos suportados pelo sistema que está sendo contado. Em resumo a fronteira separa o sistema, sob avaliação, das aplicações externas.

\section{Etapa III - Contagem de Pontos de Função não Ajustados}

Reflete o conjunto de funções disponibilizadas ao usuário, e o resultado da contagem pode ser considerado como pontos de função brutos, face à necessidade de se observar outras variáveis que influenciam nos cuidados e esforços a serem despendidos durante o processo de desenvolvimento do sistema. Assim as funções são definidas como:

- Arquivos Lógicos Internos

- Arquivos de Interface Externa

- Entradas Externas

- Saídas Externas

- Consultas Externas

\section{Regras para Contagem dos Pontos de Função Brutos}

Após a definição sobre o tipo de contagem que será realizada, ou seja, se o sistema a ser dimensionado é um projeto de desenvolvimento, manutenção ou produção, e após a identificação da fronteira da aplicação, segue-se à identificação dos grupos de funções tipo dados e transações, e a contagem de seus registros e itens de dados.

- Arquivo Lógico Interno:Trata-se de grupos de dados ou informações de controle ${ }^{1}$, interrelacionados, requisitados pelo usuário como necessidades de informação, cuja

\footnotetext{
${ }^{1}$ Informação de controle é o dado utilizado pelo sistema para garantir que todas as funções sejam realizadas conforme solicitado pelo usuário.
} 
manutenção, realizada por um processo elementar $^{2}$ da aplicação(alteração, inclusão, exclusão), acontece dentro da fronteira da aplicação.

\section{Complexidade Funcional dos ALI}

A complexidade é determinada em função da quantidade de registros lógicos e itens de dados referenciados.

\begin{tabular}{|c|l|c|c|}
\hline & $\begin{array}{l}\text { 1 a 19 } \\
\text { Itens de } \\
\text { Dados }\end{array}$ & $\begin{array}{l}\text { 20 a } 50 \text { Itens } \\
\text { de Dados }\end{array}$ & $\begin{array}{l}\text { 51 ou mais Itens } \\
\text { de Dados }\end{array}$ \\
\hline $\begin{array}{c}\text { 1 Registro } \\
\text { Lógico }\end{array}$ & $\{$ SIMPLES & $\{$ SIMPLES $\}$ & $\{\mathrm{MÉDIA}\}$ \\
\hline $\begin{array}{c}\text { 2 a } 5 \text { Registro } \\
\text { Lógico }\end{array}$ & $\{$ SIMPLES $\}$ & $\{\mathrm{MÉDIA}\}$ & $\{\mathrm{COMPLEXA}\}$ \\
\hline $\begin{array}{c}\text { 6 ou mais } \\
\text { Registro } \\
\text { Lógico }\end{array}$ & $\{\mathrm{MÉDIA}\}$ & $\{$ COMPLEXA $\}$ & $\{\mathrm{COMPLEXA}\}$ \\
\hline
\end{tabular}

Tab 2- Matriz de amostra para Determinação do Nível de Complexidade do Processamento da

Informação para cada Arquivo Lógico Interno ALI

\begin{tabular}{|l|l|}
\hline Complexidade & pontos de função \\
\hline Simples & 7 \\
\hline Média & 10 \\
\hline Complexa & 15 \\
\hline
\end{tabular}

Tab 3 : Contribuição dos ALI para a contagem dos pontos de função brutos

arquivo de Interface Externa (AIE): São grupos de dados, inter-relacionados, necessários para a aplicação, mantidos e armazenados fora do sistema que está sendo dimensionado. Baseia-se na Identificação dos dados armazenados fora da fronteira da aplicação, dados que não sofrem manutenções pela aplicação que está sendo avaliada, dados identificados como necessidades de informação do usuário e dos dados que são contados como ALI para outra aplicação. A complexidade é determinada em função da quantidade de registros lógicos e itens de dados referenciados.

\begin{tabular}{|l|l|l|l|}
\hline & $\begin{array}{c}\text { 1 a 19 } \\
\text { Itens de } \\
\text { Dados }\end{array}$ & $\begin{array}{c}\text { 20 a } 50 \text { Itens } \\
\text { de Dados }\end{array}$ & $\begin{array}{c}\text { 51 ou mais } \\
\text { Itens de } \\
\text { Dados }\end{array}$ \\
\hline 1 Registro lógico & $\{$ SIMPLES & $\{$ SIMPLES $\}$ & $\{$ MÉDIA $\}$ \\
\hline 2 a 5 Registro lógicos & $\{$ SIMPLES $\}$ & $\{$ MÉDIA $\}$ & $\{$ COMPLEXA $\}$ \\
\hline $\begin{array}{l}\text { 6 ou mais Registros } \\
\text { lógicos }\end{array}$ & $\{$ MÉDIA $\}$ & $\{$ COMPLEXA $\}$ & $\{$ COMPLEXA $\}$ \\
\hline
\end{tabular}

Tab 4 - Matriz de amostra para Determinação do Nível de Complexidade do Processamento da Informação para Arquivo Interface Externa.

\begin{tabular}{|l|l|}
\hline Complexidade & pontos de função \\
\hline Simples & 5 \\
\hline Média & 7 \\
\hline Complexa & 10 \\
\hline
\end{tabular}

Tab 5 -Contribuição dos AIE para a contagem dos pontos de função brutos

\footnotetext{
${ }^{2}$ Processo elementar é menor atividade percebida pelo usuário que dever ser realizada pelo sistema
}

- Entradas Externas - EE: São grupos de dados que entram no sistema, utilizados para a manutenção dos Arquivos Lógicos Internos, ou seja, provocam uma inclusão, exclusão e/ou alteração nos dados dos ALI. Baseia-se na Identificação dos processos que recebem dados externos e que atualizam arquivos lógicos internos. A complexidade funcional da EE é determinada em função da quantidade de arquivos lógicos internos e itens de dados referenciados.

\begin{tabular}{|l|l|l|l|}
\hline & $\begin{array}{c}1 \text { a } 4 \text { Itens de } \\
\text { Dados }\end{array}$ & $\begin{array}{c}\text { 5 a 15 Itens } \\
\text { de Dados }\end{array}$ & $\begin{array}{c}16 \text { ou mais } \\
\text { Itens de Dados }\end{array}$ \\
\hline $\begin{array}{l}\text { 0 a 1 Arq. lógicos } \\
\text { Referenciados }\end{array}$ & $\{$ SIMPLES & $\{$ SIMPLES $\}$ & $\{$ MÉDIA $\}$ \\
\hline $\begin{array}{l}\text { 2 Arq. lógicos } \\
\text { Referenciados }\end{array}$ & $\{$ SIMPLES $\}$ & $\{$ MÉDIA $\}$ & $\{$ COMPLEXA $\}$ \\
\hline $\begin{array}{l}\text { 3 ou mais Arq. lógicos } \\
\text { referenciados }\end{array}$ & $\{$ MÉDIA $\}$ & $\{$ COMPLEXA $\}$ & $\{$ COMPLEXA $\}$ \\
\hline
\end{tabular}

Tab 6 - Matriz de amostra para Determinação do Nível de Complexidade do Processamento da Informação para Entradas Externas.

\begin{tabular}{|l|l|}
\hline Complexidade & pontos de função \\
\hline Simples & 3 \\
\hline Média & 4 \\
\hline Complexa & 6 \\
\hline
\end{tabular}

Tab 7 -Contribuição dos EE para a contagem dos pontos de função brutos

- Saídas Externas - SE: representam as atividades do sistema que transformam dados dos arquivos lógicos internos e geram resultados que são exibidos ao usuário. A contagem das saídas externas é baseada na quantidade de arquivos lógicos referenciados e na quantidade de itens de dados referenciados. A Identificação da Saídas Externas - SE baseia-se nos processos que enviam dados para fora da fronteira da aplicação. Contando-se cada processo que envia dados ou informações para fora da fronteira da aplicação, bem como cada processo que necessitar de lógica de processamento $^{3}$ diferente da utilizada em outras saídas externas que tenham o mesmo formato. A complexidade funcional das SE é determinada em função da quantidade de arquivos lógicos e itens de dados referenciados.

\begin{tabular}{|c|l|l|l|}
\hline & $\begin{array}{c}1 \text { a } 4 \text { Itens de } \\
\text { Dados }\end{array}$ & $\begin{array}{c}5 \text { a 15 Itens } \\
\text { de Dados }\end{array}$ & $\begin{array}{c}16 \text { ou mais } \\
\text { Itens de } \\
\text { Dados }\end{array}$ \\
\hline $\begin{array}{c}\text { 0 a 1 Arquivos lógicos } \\
\text { Referenciados }\end{array}$ & $\{$ SIMPLES $\}$ & $\{$ SIMPLES $\}$ & $\{$ MÉDIA $\}$ \\
\hline $\begin{array}{c}\text { 2 a 3 Arquivos lógicos } \\
\text { Referenciados }\end{array}$ & $\{$ SIMPLES $\}$ & $\{$ MÉDIA $\}$ & $\{$ COMPLEXA $\}$ \\
\hline 4 ou mais Arquivos & & & \\
\hline
\end{tabular}

\footnotetext{
${ }^{3}$ Lógica de processamento é o conjunto de procedimentos de conferência, cálculos, referência e acessos, solicitados pelo usuário.
} 
\begin{tabular}{|l|l|l|l|}
\hline lógicos referenciados & $\{$ MÉDIA & \{COMPLEXA & \{COMPLEXA \\
\hline
\end{tabular}

Tab 8- Matriz de amostra para Determinação do

Nível de Complexidade do Processamento da Informação para Saídas Externas.

\begin{tabular}{|l|l|}
\hline Complexidade & pontos de função \\
\hline Simples & 4 \\
\hline Média & 5 \\
\hline Complexa & 7 \\
\hline & \\
\hline
\end{tabular}

Tab 9 - Contribuição dos SE para a contagem dos pontos de função brutos

- Consultas Externas - CE : São requisições de informações que, para serem satisfeitas, precisam que sejam combinados parâmetros de entradas e saídas que permitem a recuperação da informação solicitada pelo usuário. Nenhum arquivo lógico interno é mantido durante o processo, e as informações que compõem a saída não são dados derivados, ou seja, correspondem, exatamente, aos dados solicitados, na forma em que estiverem armazenados nos arquivos lógicos internos. Baseia-se na Identificação dos Processos onde uma entrada está associada a uma recuperação e exibição de dados, sem que haja processamentos, ou seja, não acontece transformações dos dados para serem exibidos aos usuários. São contados os arquivos lógicos e itens de dados referenciados deverão ser contados, considerando as entradas e saídas, separadamente, bem como cada processo de recuperação de dados que seleciona dados com base em parâmetros de entrada fornecidos.

Complexidade funcional das CE A complexidade é determinada em função da quantidade de arquivos lógicos e itens de dados referenciados, observando-se separadamente, as entradas e saídas.

\begin{tabular}{|l|l|l|l|}
\hline & $\begin{array}{l}1 \text { a } 4 \text { Itens } \\
\text { de Dados }\end{array}$ & $\begin{array}{l}5 \text { a } 15 \text { Itens de } \\
\text { Dados }\end{array}$ & $\begin{array}{l}16 \text { ou mais } \\
\text { tens de Dados }\end{array}$ \\
\hline $\begin{array}{l}\text { 1 Arq.lógico } \\
\text { Referenciado }\end{array}$ & $\begin{array}{l}\{\text { SIMPLES } \\
\}\end{array}$ & $\{$ SIMPLES $\}$ & $\{$ MÉDIA $\}$ \\
\hline $\begin{array}{l}\text { 2 Arq. lógicos } \\
\text { Referenciados }\end{array}$ & $\{$ SIMPLES & $\{$ MÉDIA $\}$ & $\{$ COMPLEXA $\}$ \\
\hline $\begin{array}{l}3 \text { ou mais Arq. lógicos } \\
\text { referenciados }\end{array}$ & $\{$ MÉDIA $\}$ & $\{$ COMPLEXA $\}$ & $\{$ COMPLEXA $\}$ \\
\hline
\end{tabular}

Tab 10 - Matriz de amostra para Determinação do Nível de Complexidade do Processamento da informação para Entradas Externas - Parte da consulta correspondente às Entradas

\begin{tabular}{|l|l|l|l|}
\hline & $\begin{array}{c}\text { 1 a } 5 \text { Itens } \\
\text { de Dados }\end{array}$ & $\begin{array}{c}6 \text { a 19 Itens } \\
\text { de Dados }\end{array}$ & $\begin{array}{c}\text { 20 ou mais } \\
\text { Itens de } \\
\text { Dados }\end{array}$ \\
\hline $\begin{array}{l}\text { 1 Arq. lógico } \\
\text { Referenciado }\end{array}$ & $\{$ SIMPLES $\}$ & $\{$ SIMPLES $\}$ & $\{\mathrm{MÉDIA}\}$ \\
\hline $\begin{array}{l}\text { 2 a 3 Arq. lógicos } \\
\text { Referenciados }\end{array}$ & $\{$ SIMPLES $\}$ & $\{\mathrm{MÉDIA}\}$ & $\{\mathrm{COMPLEXA}\}$ \\
\hline $\begin{array}{l}\text { 4 ou mais Arq. } \\
\text { lógicos } \\
\text { referenciados }\end{array}$ & $\{\mathrm{MÉDIA}\}$ & $\{\mathrm{COMPLEXA}\}$ & $\{\mathrm{COMPLEXA}\}$ \\
\hline
\end{tabular}

Tab 11 - Matriz de amostra para Determinação do Nível de Complexidade do Processamento da
Informação para Consultas Externas - Parte da consulta correspondente às Saída

\begin{tabular}{|l|l|}
\hline Complexidade & pontos de função \\
\hline Simples & 3 \\
\hline Média & 4 \\
\hline Complexa & 6 \\
\hline
\end{tabular}

Tab 12 - Contribuição das CE para a contagem dos pontos de função brutos

\section{Cálculo de Pontos de Função não Ajustados ou Brutos}

Após a definição da fronteira da aplicação, da escolha do tipo de contagem, da agregação das funções de acordo com as cinco categorias de funções e da classificação da complexidade, podemos calcular os pontos de função não ajustados, ou brutos, aplicando as regras contidas na seguinte tabela:

\begin{tabular}{|c|c|c|c|}
\hline Descrição & Complexidade funcional & $\begin{array}{l}\text { Total } \\
\text { complex }\end{array}$ & $\begin{array}{l}\text { Total } \\
\text { por Tipo } \\
\text { função }\end{array}$ \\
\hline $\begin{array}{l}\text { Arquivo } \\
\text { lógico } \\
\text { Interno }\end{array}$ & $\begin{array}{ll}\text { (Qt Arq. Complex. simples) X } 7 \\
\text { (Qt.Arq. complex média) } & \times 10 \\
\text { (Qt. Arq. Complexas) } & \times 15\end{array}$ & & \\
\hline $\begin{array}{l}\text { Arquivo de } \\
\text { Interface } \\
\text { Externa }\end{array}$ & $\begin{array}{l}\text { (Qt. Arq. Complex simples)X } 5 \\
\text { (Qt. Arq. Complex média ) X } 7 \\
\text { (Qt Arq. Complexas) } \\
\text { X } 10\end{array}$ & & \\
\hline $\begin{array}{l}\text { Entrada } \\
\text { Externa }\end{array}$ & $\begin{array}{l}\text { (Qt. Entradas complex simples) X } 3 \\
\text { (Qt. Entradas complex média) } \times 4 \\
\text { (Qt. Entradas complexas) }\end{array}$ & & \\
\hline $\begin{array}{l}\text { Saída } \\
\text { Externa }\end{array}$ & $\begin{array}{lll}\text { (Qt. Saídas } & \text { complex simples) } \times 4 \\
\text { (Qt. Saídas } & \text { complex média) } & \times 5 \\
\text { (Qt. Saídas } & \text { complexas) } & \times 7\end{array}$ & & \\
\hline $\begin{array}{l}\text { Consulta } \\
\text { Externa (*) }\end{array}$ & $\begin{array}{l}\text { (Qt Consultas complex simples) } \times 3 \\
\text { (Qt. Consultas complex média) } \\
\text { (Qt. Consultas complexas) } \\
6\end{array}$ & & \\
\hline \multicolumn{2}{|l|}{ Totais } & T2 & T3 \\
\hline
\end{tabular}

(*) analisar separadamente as quantidades de arquivos e itens de dados referenciados e considerar a complexidade da consulta como sendo a maior complexidade observada entre as partes das entradas e saídas da consulta.

Tab 13 - Regras para calculo de PF Brutos

\section{Etapa IV - Cálculo do Fator de Ajuste}

A metodologia de pontos de função considera que outros fatores afetam o tamanho funcional de um sistema. Estes fatores estão relacionados com características da aplicação:

Observações:

- O nível de influência de cada uma das 14 características, a serem observadas $<$ varia de 0 a 5, correspondendo a uma escala de influência que parte da hipótese de nenhuma influência(0) até o grau de influência máxima (5)

- As características gerais do sistema podem influenciar no seu tamanho variando no intervalo de $-\mathbf{3 5 \%}$ a $\mathbf{+ 3 5 \%}$. Isto implica em um intervalo de variação para o fator de ajuste da ordem de $\mathbf{0 , 6 5}$ a 1,35. O fator de ajuste é responsável pela correção das distorções da etapa anterior. Baseia-se nas características gerais do sistema, correlacionando-as com uma tabela de 
referência que possui 14 itens, e determina o valor do nível de influência de cada item no dimensionamento do sistema.

- Processo de cálculo: avaliar o impacto de cada uma das 14 características em relação ao sistema que está sendo avaliado, atribuindo pontuação de 0 a 5 para cada característica. Calcular o nível de influência através da soma dos pontos obtidos em cada uma das 14 características. Aplicar a seguinte fórmula:

$$
\text { Fator de Ajuste }=(\mathrm{NI} * 0,01)+0,65
$$

onde: $\mathrm{NI}=$ somatório $\mathrm{da}$ pontuação atribuída a cada uma das 14 característica, refletindo o nível de Influência global no dimensionamento do sistema.

- 1 Características gerais do sistema:

1 Comunicação de dados - Grau de influência variando de 0 a 5 . : os aspectos relacionados aos recursos utilizados para a comunicação de dados do sistema deverão ser descritos de forma global.Descrever se a aplicação utiliza protocolos ${ }^{4}$ diferentes para recebimento/envio das informações do sistema.

\section{Pontuação}

0 Aplicação batch ou funciona stand-alone;

1 Aplicação batch, mas utiliza entrada de dados ou impressão remota

2 Aplicação batch, mas utiliza entrada de dados e impressão remota;

3 Aplicação com entrada de dados on-line para alimentar processamento batch ou sistema de consulta;

4 Aplicação com entrada de dados on-line, mas suporta apenas um tipo de protocolo de comunicação;

5 Aplicação com entrada de dados on-line e suporta mais de um tipo de protocolo de comunicação;

- 2 Funções distribuídas - Grau de influência variando de 0 a 5 : Esta característica refere-se a sistemas que utilizam dados ou

\footnotetext{
${ }^{4}$ Protocolo é um conjunto de informações que reconhecem e traduzem para um determinado padrão, informações entre dois sistemas ou periféricos permitindo intercâmbio das informações
}

processamento distribuído, valendo-se de diversas CPU.

Pontuação

0 Aplicação não auxilia na transferência de dados ou funções entre os processadores da empresa;

1 Aplicação prepara dados para o usuário final utilizar em outro processador (do usuário final), tal como planilhas em pc;

2 Aplicação prepara dados para transferência, transfere - os para serem processados em outro equipamento da empresa (não pelo usuário final);

3 Processamento é distribuído e a transferência de dados é online e apenas em uma direção;

4 Processamento é distribuído e a transferência de dados é online e em ambas as direções;

5 As funções de processamento são dinamicamente executadas no equipamento (CPU) mais apropriada;

- 3 Desempenho: Trata-se de parâmetros estabelecidos pelo usuário como aceitáveis, relativos a tempo de resposta.

\section{Pontuação}

0 Nenhum requerimento especial de desempenho foi solicitado pelo usuário:

1 Requerimentos de desempenho foram estabelecidos e revistos, mas nenhuma ação especial foi requerida;

2 Tempo de resposta e volume de processamento são itens críticos durante horários de pico de processamento. Nenhuma determinação especial para a utilização do processador foi estabelecida. A data limite para a disponibilidade de processamento é sempre o próximo dia útil;

3 Tempo de resposta e volume de processamento são itens críticos durante todo o horário comercial. Nenhuma determinação especial para a utilização do processador foi estabelecida. A data-limite necessária para a comunicação com outros sistemas é limitante.

4 Os requerimentos de desempenho estabelecidos 
requerem tarefas de análise de desempenho na fase de planejamento e análise da aplicação.

5 Além do descrito no item anterior, ferramentas de análise de desempenho foram usadas nas fases de planejamento, desenvolvimento e/ou implementação para atingir os requerimentos de desempenho estabelecidos pelos usuários.

- 4 Utilização do equipamento: Trata-se de observações quanto ao nível de utilização de equipamentos requerido para a execução do sistema. Este aspecto é observado com vista a planejamento de capacidades e custos. Pontuação
0 Nenhuma restrição operacional explícita ou mesmo implícito foi incluída.

1 Existem restrições operacionais leves. Não é necessário esforço especial para atender às restrições.

2 Algumas considerações de ajuste de desempenho e segurança são necessárias.

3

São necessárias especificações especiais de processador para um módulo específico da aplicação.

4

Restrições operacionais requerem cuidados especiais no processador central ou no processador dedicado para executar a aplicação.

5 Além das características do item anterior, há considerações especiais que exigem utilização de ferramentas de análise de desempenho, para a distribuição do sistema e seus componentes, nas unidades processadoras.

- 5 Volume de transações: Consiste na avaliação do nível de influência do volume de transações no projeto, desenvolvimento, implantação e manutenção do sistema.

Pontuação

0 Não estão previstos períodos de picos de volume de transação.

1 Estão previstos picos de transações mensalmente, trimestralmente, anualmente ou em certo período do ano.

2 São previstos picos semanais.

3 São previstos picos diários.

4 Alto volume de transações foi estabelecido pelo usuário, ou o tempo de resposta necessário atinge nível alto o suficiente para requerer análise de desempenho na fase de projeto.

5 Além do descrito no item anterior, é necessário utilizar ferramentas de análise de desempenho nas fases de projeto, desenvolvimento e/ou implantação.

- 6 Entrada de dados on-line : A análise desta característica permite quantificar o nível de influência exercida pela utilização de entrada de dados no modo on-line no sistema.

Pontuação

0 Todas as transações são processadas em modo batch.

1 De 1\% a 7\% das transações são entradas de dados on-line

2 De $8 \%$ a $15 \%$ das transações são entradas de dados on-line

3 De $16 \%$ a $23 \%$ das transações são entradas de dados on-line

4 De $24 \%$ a $30 \%$ das transações são entradas de dados on-line

5 Mais de $30 \%$ das transações são entradas de dados on-line

- 7 Interface com o usuário: a análise desta característica permite quantificar o grau de influência relativo aos recursos implementados com vista a tornar o sistema amigável permitindo incrementos na eficiência e satisfação do usuário final, tais como:

- Auxílio à navegação (teclas de função,acesso direto e menus dinâmicos)

a Menus

口 Documentação e help on-line

- Movimento automático do cursor.

- Movimento horizontal e vertical de tela.

- Impressão remota (via transações on-line)

- Teclas de função preestabelecidas.

- Processos batch submetidos a partir de transações on-line

- Utilização intensa de campos com vídeo reverso, intensificados, sublinhados, coloridos e outros indicadores.

- Impressão da documentação das transações on-line através de Hard copy

- Utilização de mouse

- Menus pop-up

- O menor número possível de telas para executar as funções de negócio.

- Suporte bilingüe ( contar como 4 itens)

- Suporte multilíngüe. (contar como 6 itens) 


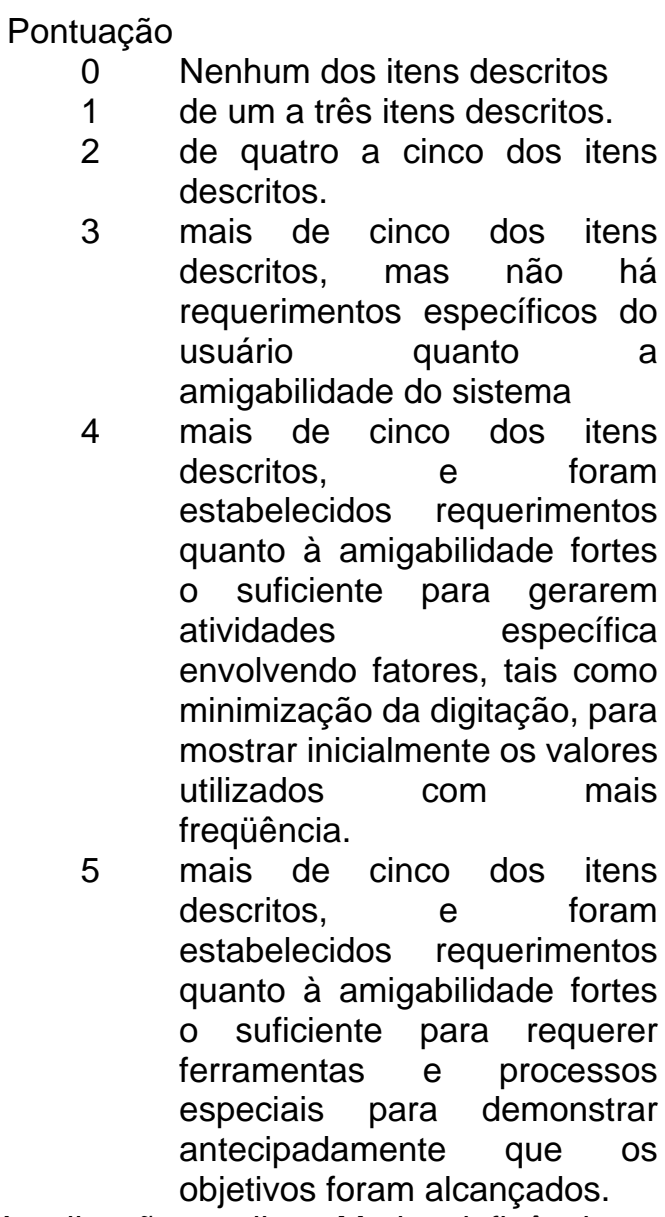

- 8 Atualizações on-line : Mede a influência no desenvolvimento do sistema face a utilização de recursos que visem a atualização dos Arquivos Lógicos Internos, no modo on-line. Pontuação

0 Nenhuma.

1 Atualização on-line de um a três arquivos lógicos internos. O volume de atualização é baixo e a recuperação de dados é simples.

2 Atualização on-line de mais de três arquivos lógicos internos. O volume de atualização é baixo e a recuperação dos dados é simples.

3 Atualização on-line da maioria dos arquivos lógicos internos.

$4 \quad$ Em adição ao item anterior, é necessário proteção contra perdas de dados que foi projetada e programada no sistema.

$5 \quad$ Além do item anterior, altos volumes trazem considerações de custo no processo de recuperação. Processos para automatizar a recuperação foram incluídos minimizando a intervenção do operador.

- 9 Processamento complexo: a complexidade de processamento influencia no dimensionamento do sistema, e portanto deve ser quantificado o seu grau de influência, com base nas seguintes categorias: processamento especial de auditoria e/ou processamento especial de segurança foram considerados na aplicação;Processamento lógico extensivo; Processamento matemático extensivo; Processamento gerando muitas exceções, resultando em transações incompletas que devem ser processadas novamente. Exemplo transações de auto-atendimento bancário interrompidas por problemas de comunicação ou com dados incompletos; Processamento complexo para manusear múltiplas possibilidades de entrada/saída. Exemplo: multimídia.

\section{Pontuação}

$0 \quad$ Nenhum dos itens descritos

1 Apenas um dos itens descritos

2 Dois dos itens descritos

3 Três dos itens descritos

4 Quatro dos itens descritos

5 Todos os cinco itens descritos.

- 10 Reusabilidade : a preocupação com o reaproveitamento de parte dos programas de uma aplicação em outras aplicações, implica em cuidados com padronização. O grau de influência no dimensionamento do sistema é quantificado observando-se os seguintes aspectos.

Pontuação

0 Nenhuma preocupação com reutilização de código.

1 Código reutilizado foi usado somente dentro da aplicação

2 Menos de 10\% da aplicação foi projetada prevendo utilização posterior do código por outra aplicação.

$310 \%$ ou mais da aplicação foi projetada prevendo utilização posterior do código por outra aplicação.

4 A aplicação foi especificamente projetada e/ou documentada para ter seu código reutilizado por outra aplicação e a aplicação é customizada pelo usuário em nível de código -fonte.

5 A aplicação foi especificamente projetada e/ou documentada para Ter seu código facilmente reutilizado por outra aplicação e a plicação é customizada para uso através de parâmetros que podem ser alterados pelo usuário. 
- 11 Facilidade de implantação : a quantificação do grau de influência desta característica é medido, observando-se o plano de conversão e implantação e/ou ferramentas utilizadas durante a fase de testes do sistema.

\section{Pontuação}

Nenhuma consideração especial foi estabelecida pelo usuário e nenhum procedimento especial é requerido na implantação.

1 Nenhuma consideração especial foi estabelecida pelo usuário, mas procedimentos especiais são necessários na implentação.

2 Requerimentos de conversão e implantação foram estabelecidos pelo usuário e roteiro de conversão e implantação foram providos e testados. O impacto da conversão no projeto nào é considerado importante.

3 Requerimentos de conversão e implantação foram estabelecidos pelo usuário e roteiro de conversão e implantação foram providos e testados. O impacto da conversão no projeto é considerado importante.

4 Além do item 2, conversão automática e ferramentas de implantação foram providas e testadas.

5 Além do item 3, conversão automática e ferramentas de implantação foram providas e testadas.

- 12 Facilidade operacional : a análise desta característica permite quantificar o nível de influência na aplicação, com relação à procedimentos operacionais automáticos que reduzem os procedimentos manuais, bem como, mecanismos de inicialização, salva e recuperação, verificados durante os testes do sistema.

Pontuação

0 Nenhuma consideração especial de operação, além do processo normal de salva foi estabelecido pelo usuário.

1-4 Verifique quais das seguintes afirmativas podem ser identificadas na aplicação . Selecione as que forem aplicadas. Cada item vale um ponto, exceto se definido explicitamente.
- Foram desenvolvidos processos de inicialização, salva e recuperação, mas a intervenção do operador é necessária.

- Foram estabelecidos processos de inicialização, salva e recuperação, e nenhuma intervenção do operador é necessária (conte como dois itens)

- A aplicação minimiza a necessidade de montar fitas magnéticas.

- A aplicação minimiza a necessidade de manuseio de papel.

5 A aplicação foi desenhada para trabalhar sem operador, nenhuma intervenção do operador é necessária para operar o sistema além de executar e encerrar a aplicação. A aplicação possui rotinas automáticas para recuperação em caso de erro.

- 13 Múltiplos locais : esta característica consiste na observação da arquitetura do projeto, observando-se a necessidade de instalação do sistema em diversos lugares.

Pontuação

0 Os requerimentos do usuário não consideraram a necessidade de instalação em mais de um local.

1 A necessidade de múltiplos locais foi considerada no projeto, e a aplicação foi desenhada para operar apenas em ambiente de software e hardware, idênticos.

2 A necessidade de múltiplos locais foi considerada no projeto, e a aplicação está preparada para trabalhar apenas em ambientes similares de software e hardware

3 A necessidade de múltiplos locais foi considerada no projeto, e a aplicação está preparada para trabalhar sob diferentes ambientes de hardware e/ou software.

4 Plano de documentação e manutenção foram providos e testados para suportar a aplicação em múltiplos locais, 
além disso, os itens 1 ou 2 caracterizam a aplicação.

Plano de documentação e manutenção foram providos e testados para suportar a aplicação em múltiplos locais, além disso, 0 item 3 caracteriza a aplicação.

- 14 Facilidade de mudanças (flexibilidade): focaliza a preocupação com a influencia da manutenção no desenvolvimento do sistema. Esta influência deve ser quantificada baseando na observação de atributos, tais como: disponibilidade de facilidades como consultas e relatórios flexíveis para atender necessidades simples. (conte como 1 item); disponibilidade de facilidades como consultas e relatórios flexíveis para atender necessidades de complexidade média (conte como 2 itens); disponibilidade de facilidades como consultas e relatórios flexíveis para atender necessidades complexas (conte 3 itens); se os dados de controle são armazenados em tabelas que são mantidas pelo usuário através de processos on-line, mas mudanças têm efeitos somente no dia seguinte; se os dados de controle são armazenados em tabelas que são mantidas pelo usuário através de processos on-line, as mudanças têm efeito imediatamente (conte como 2 itens)

Pontuação

$\begin{array}{ll}0 & \text { Nenhum dos itens descritos } \\ 1 & \text { Um dos itens descritos } \\ 2 & \text { Dois dos itens descritos } \\ 3 & \text { Três dos itens descrito } \\ 4 & \text { Quatro dos itens descritos } \\ 5 & \text { Todos os cinco itens descritos }\end{array}$

$\begin{array}{ll} & \text { Grau Descrição } \\ 0 & \text { Nenhuma influência } \\ 1 & \text { Influência mínima } \\ 2 & \text { Influência moderada } \\ 3 & \text { Influência média } \\ 4 & \text { Influência significante } \\ 5 & \text { Influência forte }\end{array}$

\begin{tabular}{||ll||l||}
\hline \hline $\begin{array}{c}\text { Características gerais do } \\
\text { sistema }\end{array}$ & $\begin{array}{l}\text { Nível de } \\
\text { influência } \\
\text { (pontuação) }\end{array}$ \\
\hline \hline 1 & Comunicação de dados & \\
\hline 2 & Funções Distribuídas & \\
\hline 3 & Desempenho & \\
\hline 4 & Configuração de equipamentos & \\
\hline 5 & Volume de transações & \\
\hline 6 & Entrada de dados On-line & \\
\hline 7 & Interface com o usuário & \\
\hline 8 & Atualização on-line & \\
\hline 9 & Processamento complexo & \\
\hline 10 & Reusabilidade & \\
\hline
\end{tabular}

\begin{tabular}{|l|l|}
\hline 11 Facilidade de implantação & \\
\hline 12 Facilidade operacional & \\
\hline 13 Múltiplos locais & \\
\hline $14 \quad \begin{array}{l}\text { Facilidade de mudanças } \\
\text { flexibilidade) }\end{array}$ & \\
\hline \hline $\begin{array}{l}\text { Somatório dos níveis de Influência }= \\
\sum N I\end{array}$ & \\
\hline Fator de Ajuste $=\left(\sum \mathrm{NI} * 0,01\right)+0,65$ & \\
\hline \hline
\end{tabular}

$\sum \mathrm{NI}=$ soma total dos graus de influência das 14 características.

Tab 14 Tabela para cálculo do nível de influência

A soma total para o ajuste de complexidade de processamento é chamada de grau de influência total que é utilizado no cálculo do total de pontos de função ajustado. O processo de ajuste de complexidade é a base de toda correção da medição. O tamanho final do ponto de função é calculado através da multiplicação dos pontos de função não - ajustados pelo fator de ajuste. Estes indicadores fornecem o grau de influência das 14 características analisadas, que o fator de ajuste calculado reflete no sistema.

\section{Etapa V - Contagem de Pontos de Função Ajustados}

Trata-se do processo que realiza a Correção das possíveis distorções acometidas durante o cálculo dos pontos de função não ajustados, aproximando as medidas à situação real.

- Cálculo de pontos de função ajustados de um projeto de desenvolvimento

PF-Desenvolvimento $=[(P F-$ Não-Ajustados + Pontos de função adicionados pelo processo de conversão) X (fator de ajuste)] (2)

- Cálculo de pontos de função ajustados de um projeto de manutenção : a contagem dos pontos de função na manutenção de sistemas é levemente diferente da contagem de ponto de função no esforço de desenvolvimento de um novo sistema. Os cinco tipos de função: arquivo lógico interno e arquivo de interface externa e entrada externa, saída externa, consulta externa, são, também, considerados na manutenção. Além disso, o número de arquivos referenciados, de itens de dados, de registros lógicos e os relacionamentos com outros sistemas e arquivos são, também, contados e ajustados a partir da atribuição de pesos como nas matrizes de complexidades descritas anteriormente. Considerando que o sistema já esteja instalado, entretanto, para cada tipo de função, os desenvolvedores de sistemas devem determinar se uma nova função está sendo adicionada ao sistema 
e/ou se uma função existente está sendo modificada ou atualizada. A medida do tamanho do ponto de função de melhorias do sistema é diferente da medida do ponto de função de esforço de desenvolvimento de sistema. Os cinco tipos de função, listados acima, ainda são considerados. São contadas cada nova função que passa a existir e cada função que deixa de existir. $O$ valor da melhoria, ou da correção (ex: o número de pontos de função afetados) é determinado pela multiplicação do número de pontos de função não ajustado pelo ajuste de complexidade do processamento.

- Cálculo de pontos de função ajustados de uma aplicação (sistemas em produção) : este tipo de contagem é utilizado tanto no caso de dimensionamento de sistemas já implantados, no início de implantação da técnica de análise de pontos de função, quanto na situação da ocorrência de um processo de manutenção. Após a realização da manutenção a contagem dos pontos de função da aplicação deve ser atualizada. O dimensionamento de uma aplicação pode ser feito a partir do resultado da contagem de pontos de função de projetos de desenvolvimento, e/ou a partir do resultado da contagem de pontos de função de um projeto de manutenção. A fórmula para 0 dimensionamento de sistemas já implantados (em produção)

- PF da aplicação =PF-Não ajustados X Fator de ajuste. (3)

Onde:

PF da aplicação : pontos de função da aplicação (tamanho funcional do sistema)

PF - Não ajustados : pontos de função não ajustados calculados para a aplicação.

Fator de ajuste : é o ajuste detectado face o cálculo dos graus de influência das 14 características que implicam no esforço e consumo de recursos para o desenvolvimento do sistema.

\section{Fórmulas}

- Fórmula para o dimensionamento de sistemas já implantados (em produção), a partir de resultado de contagem de pontos de função de projeto de desenvolvimento.

PF da aplicação = PF do Proj. Desenvolvimento (PF da conversão $X$ fator de ajuste) (4)

Onde:

PF da aplicação : total de pontos de função da aplicação.

PF do Proj. Desenvolvimento : total de pontos de função do projeto de desenvolvimento.
PF da conversão : total de pontos de função não ajustados das funções de conversão de dados no projeto de desenvolvimento.

Fator de ajuste : é o valor do ajuste detectado face o cálculo dos graus de influência das 14 características que implicam no esforço e consumo de recursos para o desenvolvimento do sistema.

a Fórmula para o dimensionamento de sistemas já implantados (em produção), a partir de resultado de contagem de pontos de função de projeto de manutenção.

$P F$ da aplicação = (PF não ajustado + PF incluído + PF alterado atual - PF alterado anterior - PF excluído ) X Fator de ajuste atual (5)

Onde:

PF da aplicação : total de pontos de função da aplicação.

PF não ajustado : total de pontos de função não ajustados da aplicação antes do projeto de manutenção

PF incluído : total de pontos de função não ajustados das funções das funções que foram incluídas na aplicação.

PF alterado atual : total de pontos de função não ajustados das funções que foram modificadas na aplicação.(mostra as funções após a modificação) PF alterado anterior : total de pontos de função não ajustados das funções que foram modificadas na aplicação (mostra as funções antes da modificação)

PF excluído : Total de pontos de função não ajustados das funções que foram removidas da aplicação.

Fator de ajuste atual : valor do ajuste detectado após o projeto de manutenção

\section{Atualização da Base de Informações. [3]}

O tamanho de um sistema em pontos de função (ou base de contagem) deve ser mantido atualizado, pois é a base para toda medição que envolva produtividade. Assim que novas capacidades forem adicionadas ao sistema, ou funções forem removidas, todo o tamanho do sistema em pontos de função deve ser modificado. Por exemplo, para um sistema entregue a usuários no final de determinado ano, por exemplo, em 1995, outras versões serão liberadas várias vezes durante o ano, ou por motivo de melhorias, correções, ou manutenções legais. Para cada versão, uma nova capacidade funcional é adicionada, funções são eliminadas e deverão ser feitas modificações no tamanho das funções existentes na base da contagem anterior às modificações. Os seguintes indicadores podem ser calculados conforme segue:

- A produtividade do desenvolvimento ou a taxa de entrega em determinada unidade de 
tempo ( quantidade de pontos de função entregues por unidade de tempo).

- A melhoria de produtividade ou taxa de mudança face às alterações realizadas na aplicação para fins de melhoria a partir de determinado período ( cálculo comparativo entre as taxas de produtividade observadas nos dois períodos de tempo).

- A produtividade da atividade de suporte ou a taxa de suporte em relação à manutenção do sistema (comparação entre as taxas de atendimento de suporte e de manutenção)

Taxa de produtividade no desenvolvimento.

A taxa de produtividade do desenvolvimento é chamada de taxa de entrega, e é calculada encontrando-se a razão entre Número de pontos de função entregues e a quantidade de unidades de tempo utilizados para produzi-los.

Fazendo um acompanhamento desta taxa na atividade de desenvolvimento de projetos, o sucesso dos sistemas pode ser medido e refletirem a aceitabilidade do programa de melhoria de produtividade adotado. É através da comparação da taxa de entrega observada em uma base regular e a taxa de modificações que poderemos identificar o sucesso do programa de melhoria adotado.

\section{Determinação da Produtividade da Manutenção.}

No ambiente de manutenção, a produtividade pode ser expressa através da razão entre o esforço requerido (ex: entrada) e o tamanho do sistema em termos de pontos de função (ex: saída). Existem duas diferentes medidas de produtividade, que apresentaremos a seguir:

Atividades de melhoria de sistema. São Iniciativas de melhoria podem ser dimensionadas por pontos de função.

A razão entre o tamanho, em pontos de função, da melhoria e o esforço de trabalho requerido para fazer a modificação é a taxa de melhoria, que é também conhecida por taxa de mudança.

O esforço relativo ao trabalho executado pode ser medido tanto por meses trabalhados como por horas ou dias trabalhados. Os desenvolvedores de sistemas devem usar uma medida consistente de forma que eles possam analisar as tendências das taxas de produtividade.

\section{Atividades de Suporte ao Desenvolvimento de Sistemas}

Para determinar suporte ao usuário, a quantidade de recursos de manutenção que está sendo expandida para atendimento aos usuários, a taxa de produtividade será a razão entre o tamanho do sistema e o esforço

\section{Análise de Resultados}

Ganho de produtividade é a diferença entre as taxas correntes de entrega, modificação, ou taxa de suporte. Depois que o ganho é calculado, analisando-se o ambiente, pode-se compreender a razão para o ganho a partir da execução de análises de pontos de função.

Neste momento, torna-se importante a análise do porquê das mudanças nas taxas ou das causas que provocam diferenças entre as taxas de projetos similares. Esta análise fornece os seguintes benefícios:

- Permite medir a dimensão das iniciativas de melhoria de produtividade incluídas nos planos de desenvolvimento. (Ex. a criação de testes de regressão).

- Permite identificar oportunidades para melhorias de produtividade em outras áreas comparando as melhores práticas, ou seja, comparando as técnicas de melhorias utilizadas que obtiveram mais sucesso, quando utilizadas em outros projetos (ex: utilização de código comum).

- Permite subsidiar tomadas de decisões de suporte, tais como gerência de quais iniciativas como a reengenharia em sistemas existentes que exigem mais tempo que a média;

Este tipo de análise comparativa de projetos similares ajuda a responder questões como as seguintes:

- A introdução de um gerador de código torna o projeto mais produtivo? Caso afirmativo, em que grau?

- É mais produtivo comprar um pacote de software durante o desenvolvimento ou realizar a compra durante a manutenção ?

- Qual é o efeito da geração de relatórios para usuário final e a reutilização de código nas taxas de produtividade globais?

Perfil de aplicação do sistema fornece a informação necessária para a execução deste tipo de análise. Este perfil, que é usado para descrever as características do processo de desenvolvimento, é critico para a análise do impacto compreensível de ferramentas específicas, técnicas, e processos que formam o programa de melhoria de produtividade. O perfil deve incluir:

a Linguagem

a Hardware

- Técnicas e ferramentas de produtividade utilizadas

- O ambiente alvo

- O ambiente de desenvolvimento

- A idade do sistema que está sendo suportado

- A experiência do grupo de desenvolvimento 
Comparando as taxas de produtividade dos projetos, o exame dos perfis dos projetos permite a análise das causas das diferenças de produtividades entre projetos. Fazendo 0 acompanhamento das taxas de modificação, pode-se medir o sucesso de tais iniciativas de melhoria de produtividade assim como a introdução de novas ferramentas.

\section{Estimação da Produtividade com Pontos de Função}

Um dos maiores benefícios da utilização da técnica de ponto de função é a possibilidade de medir o tamanho dos projetos ou melhorias e suporte para a elaboração de estimativas mais coerentes e corretas. Os parágrafos a seguir descrevem como as três taxas de entrega de produtividade, modificação e suporte podem ser utilizadas para previsão de esforços de trabalhos futuros. Podem-se fazer as primeiras estimativas do tamanho de todos os pontos de função do desenvolvimento de um projeto em particular, durante o planejamento do projeto, podendo, continuamente, refiná-lo até que o cálculo esteja completo no final do projeto detalhado. Para se estimar todo o esforço despendido em um projeto, na etapa de planejamento, o tamanho do ponto de função estimado para o desenvolvimento do projeto é dividido pela taxa de entrega atual, Assim (estes números podem ser arredondados para os últimos meses completos) esta estimativa pode, então, ser extrapolada para os meses decorridos, depois do ajuste da definição do número de pessoas alocadas no projeto.

Melhorias

Cada melhoria pode ser medida usando-se a técnica de ponto de função. Novamente podese fazer a primeira estimativa durante a fase de planejamento e refiná-lo conforme os novos detalhes vão surgindo. A estimativa pode ser derivada a partir da divisão do tamanho do ponto de função pela taxa de melhoria corrente.

Suporte

Ao estimar os requisitos de suporte, podese determinar o número de horas que serão necessárias para suportar o portifólio de sistemas e, baseado neste número calcular os recursos necessários e dimensionar a equipe de suporte. Deve-se refinar, continuamente, a estimativa conforme o tamanho do sistema, em termos de funcionalidades (ponto de função) e capacidades se aproximem daquelas que realmente irão ser implementadas. Para uma estimativa justa, é essencial que a alimentação da base de contagem e - o histórico das taxas de produtividade em outros projetos - estejam devidamente atualizadas e corretas.

Escolha da taxa de produtividade apropriada

Como última meta, a taxa de produtividade utilizada para calcular o esforço para novos trabalhos de desenvolvimento ou melhorias deveria satisfazer a entrega ou taxa de modificação que ocorre no nível do projeto, até que a taxa média para todo o departamento de sistemas, ou para a unidade apropriada dentro da área de sistemas, possa ser utilizada. Embora esta taxa não seja tão justa como taxa única por projeto, a estimativa global é, significativamente, mais correta do que outros métodos de estimação. Para estimações e comparações, é essencial que projetos e ambientes similares sejam utilizados. Por exemplo, a taxa de entrega para aplicação baseada em micro computador não deve ser usada para estimar o esforço de desenvolvimento para uma aplicação descentralizada baseada em grande porte.

Refinamento das estimativas

A taxa de produtividade usada para calcular este refinamento é baseada no conhecimento, em informações de relatório, e é incluída, certamente, nos fatores de produtividade. Por exemplo, se todos os projetos usam técnicas estruturadas 0 ganho de produtividade destas técnicas já deverá estar incluído na taxa. Novas técnicas e ferramentas, que são parte do programa de melhoria de produtividade, no entanto, não estão incluídas nas taxas. $O$ efeito global pode ser medido através da comparação entre as taxas para um projeto utilizando-se estas novas facilidades e as taxas de mercado obtidas a partir de estudos de "benchmarking".

A estimativa para projetos futuros utilizando técnicas ou ferramentas pode ser refinada através do ajustamento do ganho de produtividade esperado, assim como as novas facilidades se tornam maduras e alcançam total penetração na organização, assim, a taxa média deve ser ajustada de acordo com as metas da organização.

Gerador de Relatórios e Produtividade para o Usuário Final

Medir os efeitos de certos fatores relacionados com a produtividade de quem desenvolve sistemas é uma das conquistas do programa de métricas. Uma das chaves que mais contribuem para a melhoria da produtividade é o aumento das responsabilidades e participação do usuário final, no desenvolvimento. Existem diversas formas de medir a produtividade do desenvolvimento de sistemas. Entretanto, usando-se a técnica de análise de pontos de função, a produtividade do usuário final como desenvolvedor pode ser determinada. Interessase, basicamente, por duas medidas considerando a técnica de análise de pontos de função:

a Todos os efeitos que podem ocorrer a partir das informações dos usuários finais em relação a equipe do projeto;

- A quantidade de trabalho que o pessoal do projeto possa permitir que os usuários finais 
executem, ou seja, o nível de participação do usuário final no desenvolvimento do sistema.

A quantidade de pontos de função das aplicações desenvolvidas pelos usuários finais ou criadas pelos especialistas de negócio (exemplo: o gerente de sistemas temporariamente relacionado para fornecer suporte tecnológico para usuários finais) pode ser determinado da mesma maneira como é determinado o tamanho da aplicação em pontos de função para desenvolvedores de aplicações dentro da área de sistemas. Este número pode ser analisado em base mensal ou quadrimestral para se determinar o progresso do programa de geração de relatórios junto ao usuário final, e se necessário, a taxa de produtividade que os desenvolvedores deste tipo de aplicação poderiam alcançar.

Reutilização e Produtividade

A Reutilização provoca um impacto significativo sobre a produtividade. A medição deste impacto requer uma técnica específica dentro da análise de pontos de função.

Interessa-se em quão produtivo os projetos poderiam ser se possibilidades de reuso fossem utilizadas. Para medir o efeito de reusabilidade na produtividade, os desenvolvedores de sistemas podem comparar projetos similares e analisar a utilização de reuso de código, da seguinte forma, por exemplo: [4]

Projeto A: Taxa de entrega $=21,5$ PF por mês de trabalho mês de trabalho

Projeto B: Taxa de entrega $=29,0$ PF por

Baseado na análise, considerando que a única diferença entre estes projetos é a reutilização, o efeito na produtividade é estimado em cerca de 35\%. Este percentual difere, dependendo do grau de reusabilidade alcançado (por exemplo, a indústria informa que em $90 \%$ de reuso, o crescimento do ganho em produtividade fica em torno de 600\%). Os desenvolvedores e os especialistas em manutenção do repositório (exemplo: local de armazenamento central de dados, processo, código, diagramas e projeto) podem assumir este ganho de produtividade.

Pode-se, também, contar os pontos de função da reutilização do repositório. Devido a natureza de algum componente de reuso, o repositório entregará muitos pontos de função como um produto, os ganhos de produtividade são compreendidos pelos usuários destes recursos. Isto é possível, entretanto, para determinar o que é chamado de ponto de função nivelado para o repositório através da multiplicação do tamanho do ponto de função pelo número de usuários. Pode-se refinar este valor nivelado, considerando a porcentagem de código que os usuários selecionaram do repositório ( exemplo: os usuários que selecionaram $80 \%$ de suas aplicações do repositório possuem um valor de nivelamento maior que os usuários que apenas selecionaram um componente muito pequeno). Além disso, as taxas de entrega, também, podem diferir. Indicadores financeiros.

\section{Custo de Serviços de Informática}

Os serviços de processamento de dados podem ser segmentados em três tipos básicos: desenvolvimento, manutenção/ suporte e produção. A literatura menciona cinco principais modelos para a apuração de custos com serviços de processamento de dados, que em geral são adotados pelos bureaux de serviços. Como sendo: critério tipo Test Unit, critério tipo Pontos de função, critérios tipo soma de volumes, critérios tipo volume de registros processados, e critérios tipo block time ou hora sala. Esses modelos são utilizados, em geral, não para a apuração do custo propriamente dito, mas para a determinação de preços. [5] [6]

O resultado da aplicação de um modelo de agregação de custo deve apresentar as seguintes características:

- Estimação dos custos de um serviço de informática deve ser independente de características muito específicas de cada sistema.

- Não se deve levar em consideração a estrutura interna dos prestadores de serviços, no caso de serviços terceirizados.

- O modelo deve ser adaptável a qualquer mudança que possa ocorrer no ambiente.

- O modelo ao ser implementado, deve contemplar um módulo para simulações de custos, com a finalidade de análise de cenários, para planejamento estratégico.

\section{Considerações Sobre os Cálculos:}

Destes três segmentos o que traz maior dificuldade para a apuração é o custo com desenvolvimento. O custo deverá ser observado em relação a cada fase de desenvolvimento de um projeto, ou seja, para cada fase deverá ser acompanhado o custo dos recursos alocados para a execução com as atividades que contribuem para a realização das etapas durante o ciclo de desenvolvimento do sistema. (requisitos, análise, Desenho, documentação, implementação, testes, implantação)

Desta forma, deverão ser considerados em linhas gerais os seguintes recursos:

- Hardware: este recurso poderá ser dimensionado em função da utilização de máquinas de desenvolvimento. Deverão ser computadas, para cada projeto, tempo de processamento, tempo de $\mathrm{I} / \mathrm{O}$, tempo de espera. Estes dados poderão ser obtidos através dos LOG das máquinas utilizadas pela equipe de desenvolvimento. O cálculo do custo, propriamente dito, poderá ser realizado 
com base no preço da máquina e na sua capacidade de produção.

- Software: Todos os recursos de software utilizados deverão ser considerados, como ferramentas case, software básico, etc..O cálculo deverá corresponder a um rateio, proporcional às horas de utilização do software durante cada fase de desenvolvimento do sistema.

- Recursos Humanos : os recursos humanos deverão ser rateados, proporcionalmente às horas dedicadas ao projeto.

口 Instalações: padrão de rateio deverá ser obedecido, ou seja, deverá ser proporcional às horas de utilização para 0 desenvolvimento do sistema.

\section{Considerações Finais}

O sucesso de um programa de métricas depende de uma série de fatores. Estes variam desde a conscientização dos profissionais envolvidos de que medir horas de trabalho em termos de produtividade até a existência de recursos técnicos e humanos necessários para a manutenção e monitoramento do programa. É importante ressaltar que um programa de medição traz benefícios para o empregado à medida que em possibilita dimensionar melhor a carga de trabalho de forma a garantir a qualidade.Com esta informação o desenvolvedor poderá requerer mais recursos bem como dimensionar o valor pecuniário de seu trabalho além de estimar com mais precisão o tempo necessário para o desenvolvimento das suas tarefas. Para o empresário, este terá uma base de informações imprescindível para estimar seus custos e apreçar seus produtos, bem como estabelecer cronogramas com promessas de entrega de produtos em prazos mais factíveis. Desta forma, recomendamos alguns cuidados na hora de se estabelecer um programa de métricas com vistas a lograr sucesso. que: [5] [6]

Deve-se buscar um programa de medição

口 Tenha um "patrocinador" executivo que forneça suporte top-down;

- Forneça medidas que sejam inteligíveis e úteis para todos os níveis de gerenciamento dentro da organização;

- Seja compreendido por todos no departamento de sistemas como seu propósito e tipo de medição que ele executa;

- Antes de obter qualquer métrica, os gerentes de sistemas necessidade seguir os seguintes passos:

- Conseguir um patrocinador executivo;

- Realizar um estudo de benchmarking na área de sistemas, para identificar volume de recursos e métricas existentes.

- Desenvolver uma lista de métricas que devem ser alcançadas para prover as medições definidas nos passos anteriores e identificar as fontes das informações;

- Preparar uma apresentação dos propósitos e benefícios do programa de métricas e difundir o conceito para os gerentes da área de sistemas;

- Garantir, através de treinamentos na metodologia de pontos de função, que um especialista nesta matéria esteja disponível para consultoria interna.

- Formar especialistas em análise de pontos de função que se tornem disseminadores da técnica, dentro da empresa.

- Determinar qual a estratégia a ser adotada para contabilizar os pontos de função dos sistemas, criando um inventário de sistemas.

- Garantir que um treinamento adequado fique disponível para a equipe de desenvolvimento, tanto através de fontes externas como treinamentos com pessoal interno.

- Começar a contabilização, e mais importante, publicar os resultados regularmente de forma que eles fiquem disponíveis para toda a equipe de sistemas. Isto estabelece a credibilidade do programa de métricas.

\section{Bibliografia}

1. Manuais sobre análise de pontos de função. Unisys.

2. IFPUG " Ffunction Point Practces Manual Release 4.0. Jan/94

3. Braga, Antônio Análise de pontos de função . Infobook, RJ 96.

4. Brown, Darlene P. Et alii. Using function points to measure productivity from the user's point of view. Information management. Warren, 1990

5. Dreger, J. Brian Function Point Analysis Prentice Hall Inc. 1989

6. McGarry, John Information Tecnology software live cycle processes. Definition and application of a software process measurement framework.

\section{Contato}

Profa. Dra. Raquel Dias

e-mail: raquel_dias@directnet.com.br Consultora independente em Tecnologia da Informação, Economista, Doutora em Ciência da Informação pela UNB, mestre em informática pelo ITA, Pós Graduada em: Inteligência Competitiva pela UFRJ, Gerência Empresarial e Engenharia Econômica pelo ICAT/DF e em Informática e Análise de Sistemas pela PUC/DF. 